\title{
Turning the NARN into an ARC at the University of Bedfordshire - some reflections and comparisons
}

\author{
Arti Kumar \\ University of Bedfordshire, UK
}

This paper is a modified version of Kumar, A. (2010) 'Supporting action research as a CPD process', in Atlay, M. and Coughlin, A. (eds.) Creating communities: developing, enhancing and sustaining learning communities across the University of Bedfordshire. Luton: University of Bedfordshire, Chapter 18, p. 254. The concepts and quotes that have shaped this paper come from members of the Action Research Consortium (the ARC) at the University of Bedfordshire. My thanks go out to my colleagues who participated in the ARC and who contributed to my own research.

\section{Abstract}

In recent years I have increasingly been involved with action research at several levels (as a leader and participant on the NARN project and also on other institutional, collaborative and individual research studies). To fulfil my various responsibilities I convened and led the Action Research Consortium (ARC) at the University of Bedfordshire from June 2008 to June 2010. Evaluating the effects of this action provided a research topic and the context for my own personal and professional development, which I present in this paper as a reflective analysis. I focus on the main lessons learned and applied at a management level, through analysing the type of change ARC participants reported and discussed, and that I observed. My insights and recommendations are broadly informed by theories related to action research, Appreciative Inquiry and positive psychology approaches.

I argue here that action research can generate continuous improvements in pedagogy but achieving this ideal for all staff crucially depends on cycles of action research operating in a productive dynamic with their personal and professional development (and this could extend to students). This will not happen by chance, and has not happened as extensively as expected, even though my findings show that the opportunities offered through the ARC were motivating and beneficial for staff who engaged. For the potential impact of action 
research to be realised in the experience of practitioner-researchers and in the vision of universities, positive conditions must be created to support research-active programmes and communities, underpinned by congruent protocols and values. The recommendations I make here can help to promote and sustain an integral research culture and are therefore relevant to managers, as well as practitioners, who are doing, or thinking of embarking on, action research.

Key words: action research; Appreciative Inquiry; SOAR analysis; CPD; realising potential.

\section{Introduction and background}

If you are a reflective practitioner (Schön,1991) you will no doubt be hooked into cycles of 'plan-do-review', and you may already be involved in a process of pedagogic action research without calling it by that name, or indeed realising its full potential for personal and professional development. Comments made by experienced practitioner-researchers during interviews conducted to explore their perspectives, as part of my research project, are illuminating in this respect:

We're doing it all the time really.

...there were things we would have done anyway, but not as a project.

I see more benefit now than I did a few years ago. I know that we designed and introduced personal tutor groups from action research, even though we didn't call it that. But it is from action research that we introduced personal tutor groups because that's what the students wanted.

Such perspectives reflect my own past experience when I evaluated and re-designed modules year after year, comparing students' feedback with theories, consulting with my team and external examiners - even writing a student text and eventually a book (Kumar, 2007), but not thinking that these cycles counted as action research. Three implications arise: (1) Many academics - especially traditional positivist researchers - would argue that cycles of 'plan-do-review' should not count as research, however defined and whatever 
results they produce; (2) Clearly, however, even small-scale activity of this type can make a big difference. In some cases it has brought benefits to individual academic practice, in others it has influenced or changed whole-institution policy and practice; (3) Needed change is easier to implement when it builds on existing small-scale activity and taps into the real interests and values of practitioners rather than when it is imposed as large-scale, fundamental shifts in policy. With action research the possibilities are powerful because practitioners themselves put their research outcomes into practice.

Firstly, since perception and reality are closely and causally related, staff in management positions need to value, attribute and reward what practitioners perceive they already do and develop. However this should go further: action research that is defined, supported and conducted as 'systematic, reflective (and preferably collaborative) enquiry made public' (Stenhouse, 1981) has greater potential for turning the small-scale cycle of plan-doreview into an upward spiral of plan, design, propose a project plan, gain consent and ethics approval, collect data, reflect/evaluate/analyse, record/write/publish, disseminate, share, cascade. Literature searches and theoretical concepts add a robust dimension at any stage of the process. With this conceptual base in mind the ARC attempted to invoke the power of action research that lies in its generative capacity: to transform rather than simply inform; to accept the complexity of naturalistic settings rather than create controlled environments; and to seek understandings that will improve aspects of practice.

There is now an increasing body of literature on the methods and benefits of conducting action research. There seems to be far less research and writing on the topic of management frameworks which initiate and support those practitioners engaged in action research (Beard, 2010). It therefore seems worthwhile to write from a management perspective here, about the insights gained into the potential of a robust, structured and supported action research process, following many muddled attempts to shape directions and destinations for and with ARC members. Through this experience I infer and argue here that action research can create a culture of continuous development - both for staff and (by extension) for students and institutions. 


\section{What I set out to do}

\section{The action in my action research}

The action in my action research came about partly as a result of my involvement in the NARN project - as a leader of its Midlands regional group and also as a participant researcher. I attempted to apply and cascade lessons learned from the NARN back at the University of Bedfordshire (UoB), where they gained synergy to some extent by seeding into the fruitful ground offered by its Centre for Excellence in Teaching and Learning (CETL), aligned with my responsibilities as the CETL Associate Director. The main aim of the CETL was to integrate PDP and employability into a revised institutional curriculum (referred to as CRe8) - a new delivery model which was essentially in need of evaluation to establish its effectiveness.

CRe8 has been achieved at UoB through CETL Fellows and Associates working on individual and collaborative funded projects across all faculties, staff away-days and student involvement. A systematic review drawing on internal and sectoral good practice led to the formulation of five inter-related strands: Personalised Learning; Curriculum; Realistic Learning; Employability; and Assessment. Within CRe8, SOARing to Success is an integral process which further develops and focuses on a student-centred process of realistic and personalised learner development. SOAR provides ways of animating the dynamic relationships between Self, Opportunity, Aspirations and Results. It is incremental when scaffolded within subject curricula, providing early support and gradually withdrawing it as students progress through their personal 'zones of proximal development' (e.g. see references to Vygotsky in Newman and Newman, 2009). This method enables students to develop their skills, knowledge, attributes and experiences in a broad holistic frame, and to do this with more personal motivation, sense of direction and destination. It is underpinned by Appreciative Inquiry and positive psychology theories and practices (see Watkins and Cooperrider, 2000 - and more on this later in this paper).

The ARC initially attracted twenty six staff in June 2008 , some experienced and some novice researchers, drawn from the Centre for Personal and Career Development, Learning Resources and all faculties including Psychology, Education, Business, Performing Arts, Sports Studies, Public Health and Media, offering opportunities as summarised in Figure 1. However, only twelve staff from this group completed their projects very successfully. In the second cycle of my research, thirteen staff enrolled as students on the Postgraduate Certificate of Academic Practice (PgCAP) were drawn in 
under the ARC to conduct small-scale action research projects as one of their assignments. Four of the PgCAP students were from the British School of Osteopathy, two were Academic Liaison Librarians, three from Bio Sciences, one Practice Educator, one from Sport and Exercise Science, one from Sport Therapy and one from Education.

My interpretations are based on qualitative data gathered in the first cycle from focus group interviews with ARC members (conducted by a research assistant to increase the chances of objectivity) and content analyses of written material in the second cycle (undertaken in collaboration with a second research assistant). The data analyses draw upon reported changes, but also on our own observations, reflections and discussions on how the ARC has impacted so far on participants and on the wider academic practice at UoB. I will return to the findings from both these groups later.

\section{Figure 1. Underpinning values and explicit expectations for the ARC.}

My action: convening the Action Research Consortium (ARC) as a peersupported community of practitioner-researchers

My research: To explore and evaluate the type and extent of change that might occur through supporting action research as a PDP / CPD process

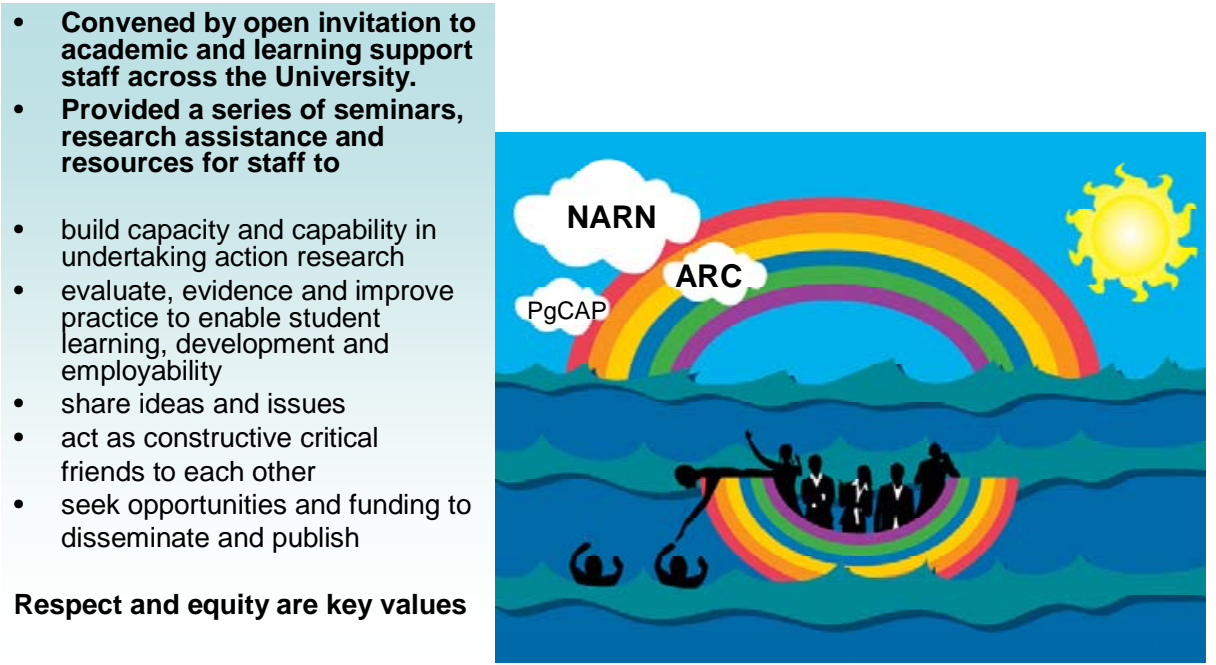

\section{Aspirations and values behind my research question}

\section{Appreciative Inquiry and a SOAR analysis}

If we wish to improve our personal and organisational efficacy, I believe we need to create and manage programmes and communities of learning designed for good and lasting effect. In doing so we need to ask questions in such a way as to 'appreciate' (increase in value) our assets, much as a house or business appreciates in value. In my case I brought 
to the ARC much prior experience and interest in developing the wider attributes of students, using Appreciative Inquiry (Watkins and Cooperrider, 2000) combined with selfassessment as an effective approach within discrete PDP and Career Development Modules, when these existed in the UoB curriculum between 1996 and 2007. There was considerable evidence from evaluations at the time that such positive psychology approaches enhanced student confidence and motivation. This gave me the impetus to develop the SOARing to Success process model and write about the conditions which can turn self-awareness into personal development plans and actions (Kumar, 2007; 2009a; 2009b).

Appreciative Inquiry can reinforce the 'want-to' and 'can-do' in the individual and the group to make a positive difference. A critical appreciation of the dynamic relationships between Self, Opportunities, Aspirations and Results was implicitly applied to the ARC as well, in the sense that action research engages Self with Opportunities such as those offered by the ARC, to tap into the Aspirations of staff (aligned with those of the HEI), producing Results in relation to self-selected aims. Ethically also it was important in my ARC project to set at rest any concerns staff may have about discovering deficits and diagnosing problems. Appreciative Inquiry helps in this respect by replacing the usual SWOT analysis that often gets bogged down in negative considerations of weaknesses and threats, with a SOAR analysis that seeks to discover and amplify what works well. With this approach one can also use what does not work simply as raw material for learning and future success.

\section{Connecting Self with Opportunity and Aspirations}

Appreciative Inquiry aligns with other values and beliefs implicit in my research:

- That most people are interested in and capable of self-development, given the right type of encouragement and supportive environment.

- That subjecting CRe8 curricula to action research would be desirable and justifiable as one way of realising the potential of e-PDP for students.

- That staff would try out approaches such as SOARing to Success (Kumar, 2007) and progressively adapt and refine them relative to their subject disciplines.

- That synergy would be generated through collaboration between peers.

The subjective nature of action research is inescapable as practitioners bring a set of personal values and expectations into the process at every stage. A key requirement for 
action research to be as ethical as possible is for practitioner-researchers to recognise and declare the reasons and values which prompt their research aims. Accordingly all ARC members were required to define the key terms and objectives of their projects, and were given opportunities in ARC meetings to refine these with peers acting as critical friends. In line with the practice and process derived from the NARN approach, templates were designed to encourage reflection and ethical considerations so that participants would declare the values, interests and experience that lay behind their research ideas, identify practical opportunities and data collection methods they could use to answer their research questions, and clarify what results they aspired to achieve.

The inclusive invitation to staff across the UoB was based on a variety of positive psychology approaches associated with personal agency and goal setting (e.g. Locke et al., 1990), and communities of learning (Senge and Scharmer, 2001). The results I aspired to achieve were aligned with an expectation expressed in our University's Education Strategy (Atlay, 2008) that all staff will engage in evidence-based and research-informed teaching practice. Also aligned with the NARN and the CETL, the ARC was envisaged as a forum for creating shared understanding around e-PDP concepts and practices, through membership of a peer-supported community that would evaluate such innovations within their subject curricula. Guidelines were issued to this effect, and for the purposes of my own research I formulated the question: 'Do ARC participants make changes to their ePDP pedagogical approaches and interventions for students as a result of the action research process?'. My earlier work in the field of personal and career development of students led me to hope we would identify collaboratively some key features of good practice in PDP and employability, and cascade the benefits to all students.

In some respects the ARC guidelines represented a departure from NARN. Action research was specified as the methodology we would all be using to investigate the processes and outcomes of interventions, for which CRe8 provided a context of changes in the content and modes of implementation of new curricula. We favoured collaboration across areas on topics of common interest: project teams were actively encouraged to include other practitioners in academic and/or learning support areas, and students as partners. 


\section{Results - what actually happened}

\section{What worked well}

The ARC offer generated twenty six applications and considerable interest, sustained at first by our common goals and values such as respect for everyone's professionalism despite diversity in the abilities, experience and disciplinary or practice backgrounds of ARC members. ARC projects have demonstrated equity and collaboration between academic staff and novice practitioner-researchers from 'Careers' and 'Learning Resources'. The core of twelve staff who persevered despite obstacles have reported considerable benefits at focus group and individual interviews. These include a renewed belief in and enthusiasm for action research, submitting articles to journals not considered before, disseminating at home and abroad, learning by doing, and discussing with others thereby enhancing research capability.

PDP requires a structured and supported process (QAA 2001, revised 2008), and the ARC set up both structure and support within a developmental set of opportunities designed to engage staff collectively and individually (Altrichter et al., 1993). Structure was provided through a shared work plan with a timeframe and collective goals, a series of organised semi-formal ARC seminars, a dedicated VLE repository for resources, and informal peer support. Two research assistants were appointed to help ARC participants with their individual proposals and plans, and especially with data collection and analysis. Feedback in focus groups shows that both this structure and the support provided has been greatly valued.

An implicit value in the ARC is that be(com)ing a reflective practitioner within a community of practice can enhance one's personal meaning-making and professional development (Wenger, 1998). My data collection methods were designed to provide opportunities for participants to reflect and plan. Open questions on templates encouraged reflective narratives to capture interim progress, to record individual learning journeys at various stages, and prompt further action. Staff could later select material from this record to use in dissemination and publication. This valuing of reflection in and on action resulted in one positive outcome: the inclusion of action research activity and assignments within the redesigned PgCAP at our university. All PgCAP students were enrolled into the second round of ARC projects. The reflective prompts in their case contributed to final written assignments which required them to be analytical about their learning and development (Kramp et al., 1995). From these reflections there is evidence of both benefits and issues: 
The action research project, as part of the PgCAP, has also helped me evaluate my own practice, but more importantly change it for the better. Although this is a formal, assessed document, I see myself continually performing internal action research, where I can identify a problem or issue, and then change my practice to try and better it.

I find the action research element of the course both challenging and interesting. Despite the steep learning curve and limited time I am very interested to see what kind of data I collect and what, if any, conclusions can be drawn.

To me, action research is personal, investigating things I have some control over and can make changes to myself, not just commenting on the current picture, but actually collaborating with others and changing the picture. As my Learning Styles Questionnaire results suggest, I am a change agent and like to experiment and try things out, so this type of research really suits me. The difficulty with this, is change takes time and this sits outside of my core job function, therefore the demands of my usual business will make it difficult to continue further cycles and make this a more developed piece of research.

It is clear from evaluations that the ARC model is actually and potentially a powerful and motivating 'structured and supported process undertaken by individuals' (such as that envisaged and defined in the PDP agenda). Why then did it not achieve wider participation and better engagement in the first cycle, with more evidence of developmental outcomes? Between the first wave of enthusiasm and later project completion rates, the numbers steadily declined. As meetings progressed through the year, if engagement is judged by attendance, then both leave much to be desired.

\section{Lessons learned from what did not work well}

I will focus here on three factors that impacted considerably on the willingness and ability of staff to engage with ARC projects. The first is related to time and timing, the second is about the importance of constructively aligned values, and the third is about the ethics approval process. 


\section{Time and timing}

Time constraints were mentioned by almost everyone in the ARC as a major reason for discontinuing projects or missing opportunities - it has been a perennial issue that the ARC could not include '36-hour wristwatches' in its otherwise generous offer. My observations on time are two-fold: firstly we need a Self-MAP (Kumar, 2007), not a watch, to manage our time. The MAP (a grounded sense of one's Motivations, Abilities and Personal styles) serves the purpose of both map and compass, helping each individual to develop a sense of destination and direction, and navigate through the project's learning journey. If we manage ourselves in this way we also get better at managing time and pressure through opportunities in learning and work. Secondly, however, the ideal of practitioners engaging in 'systematic inquiry made public' is seriously at odds with the reality of life for staff whose activity is characterised by knee-deep marking piles and hundreds of reactive interactions with a diverse body of students in rapidly changing circumstances. They are caught up in what they must do rather than what they might also do. If we wish to create learning journeys in which PDP and CPD function as cycles of action research in a productive dynamic, then we must enable these journeys as the norm - 'just the way we do things around here'.

Lack of time is similarly cited by many staff as a reason for not being able to take advantage of optional staff development workshops. Not only is there a manifest lack of engagement with such options, there is also plenty of research evidence to show that most traditional workshop methodologies fail to promote meaningful CPD that transforms pedagogic practice. For example, Joyce and Showers (2002) show that fewer than 15\% of teachers implement new ideas learned in workshops. The irony is that for staff CPD to result in more effective performance, exactly the same conditions and approaches are needed as for student PDP to be effective.

If time is important, timing is equally so - a research design and timeline must fit with a congruent ethics approval process and exploit opportunities for collecting data. This has also been an important lesson for staff that will feed into benefits for students. For example, an ARC member reported that she now appreciated how important it was to consider the time allocated for assignments and timing of research activity she was setting for students. In fact, my observations tell me that self-management, the drive for results, adaptability and using resources appropriately were key attributes in ARC project completion - just as they are for students in coping with assignments. 


\section{The importance of congruent values}

The following extracts from evaluation of the Bridges-CETL indicate how contextual factors, views and values might have influenced the priorities and ability of the CETL and ARC to achieve their objectives:

Some individual staff fully embraced Bridges' approach but getting widespread adoption of the more innovative aspects has proved more problematic. Conflicting interests and priorities get in the way. (Atlay, 2010, p.16)

It has been really difficult to achieve shared understanding because there is no accepted structure or culture to achieve this. In academia we encourage 'critical thinking' about everything - to the extent that shared thinking encouraged by a central body such as the CETL, agreement on concepts and practices etc. is almost impossible to achieve. It has been especially hard to achieve this in an area such as PDP which was initially so controversial (and may still be in some quarters). This is also to do with the traditional hierarchy of values in which we place disciplinary research first, then academic/pedagogic research, action research, teaching and learning - while PDP is a poor relation to all these. (CETL team member, taken from Facilitate evaluation, January 2009).

Despite a subtle steer towards PDP, for a variety of reasons members of the ARC chose a diverse range of projects, not always focused on PDP. Added to this, a decline in numbers of staff engaging overtly with the ARC left my initial research plan bereft of research participants (despite many having signed my consent forms). My data collection methods initially depended on staff attending meetings and completing templates. I modified my expectations to the premise that the ARC could potentially benefit its members in a variety of ways. Staff could, for example:

- Explore and evaluate any chosen aspect of their practice and pedagogy from different perspectives and experiences.

- Replicate an e-PDP process in their own CPD, as they reflect and record their personal learning journeys.

- Fulfil the university's expectations in respect of evidence-based practice, and its requirements as written into the Education Strategy and Research Strategy. 
In retrospect it has proved important to allow self-selection of project topics in this way, as evidenced by PgCAP students' comments:

Enjoy doing research, especially as I can choose the topic so it's directly relevant to my practice, and will lead to changes/improvements.

Action research has increased my ability to influence, challenge, change and improve effectiveness of clinical assessment.

My initial research question embodied a grand and ambitious idea of what I wanted to evaluate, and contained an implicit expectation that staff would use action research methodology to introduce and simultaneously evaluate PDP; that this would not only inform but transform their practice and - by extension - transform their students! I later discovered very few studies have established connections of this sort, and complex social and psychological variables make it very difficult to validly do so. For others too, in both the NARN and ARC, a significant learning point was that we had to scale back our initial grand designs to what was practicable within our circumstances and limited timeframes. We often started by describing wobbly interventions with fuzzy boundaries, which went through several iterations before becoming practically feasible and ethically sound. Learning by acting as critical friends to each other was effective and valued by all of us we challenged each other to clarify and focus on more precise definitions, intentions and actionable research aims, designs and plans. Peer support and belonging to groups also sustained our commitment in the face of complex and changing realities.

I gained a related insight about the value of allowing 'marinating time' in this process periods of muddling when nothing seems to be working or moving. It took almost the first year in many projects for feasible research questions to be framed and implemented. In my case I was naively looking for a change of culture - but this takes time and sustained effort. As John Kotter and Paul Lawrence (1974) found in their research in organisations, the more successful executives typically spent more than a year seeding and sounding out ideas with key stakeholders, gradually moving staff in a direction towards consensus in favour of a desired change. The same may be true for creating a new culture integrating action research, PDP and CPD in our university, and then evaluating the benefits of this. Through my 'marinating time' I struggled to see how I might maintain any connection with PDP. The links between PDP for students and CPD for staff are now well established 
(CRA Annual Seminar 2009: Whither CPD?), but perceiving the common ground between PDP, CPD and action research has been one of my 'aha' moments of realisation. In fact the processes involved in 'doing, reflecting, conceptualising, evaluating, planning and improving' are common to development in general - whether these are staff or student actions and interactions. Eventually I realised that I was actually already applying PDP protocols and values to the functionality of the ARC and that it would be a worthwhile project to explore the effects of this. I modified my aspirations and my research question thus: 'Can a supported action research process act as an agent of change? What type of change? Under what conditions? What works, what doesn't, and why?'.

\section{Ethics approval}

At the time of writing there is still limited hard evidence of the type of change (development/transformation) that I was initially looking for, but much has been learned about the type of support that is needed in order to bring about the desired changes. One lesson shows that the importance of aligning espoused theories with theories-in-action (Argyris and Schön,1974) is not to be underestimated. Due to the gap between the Education Strategy's espoused theories and expectations around action research and the oppositional theories-in-action of the Ethics Committee, many ARC members' aspirations were rendered impossible to implement. The following comments illustrate this well:

Ethical approval in the Education research institute is really problematic and slow. It seems impossible to do any research even though this university is striving to do more research. This should be sorted as a matter of urgency... It makes practitioner researchers feel like they are being ignored because they are not 'proper academics' and therefore this is disrespectful as well as damaging to the community of practice. (Anonymous comment from an ARC member, taken from Facilitate follow-up)

Having promoted and encouraged action research through a range of positive values and approaches in the ARC, I was concerned and frustrated about the unsympathetic and over-rigorous analysis of our project proposals, which dampened the enthusiasm of many members to engage with research. I did my best to resolve the situation and an ARC member also represented our concerns to the ethics committee, but the problem persisted. The committee asked ARC members to put their issues in writing and this is one of the responses elicited via email: 
(We) suffered terribly last year with our T\&L project when we had to submit to the education ethics committee at late notice. They wanted us to revise the proposal after T\&L/CETL had agreed it. The form filling was for PhD type activity and the length of time it took for them to agree imposed a long delay on data collection and frankly lost my interest in the whole thing. I don't think they really understood the purpose of these small scale research projects and I think for new researchers it would scare them off the whole process which would have a detrimental effect on developing a bigger research culture in the university.

When ARC project applications and proposals were submitted in the second round (200910) we were hopeful that our efforts had generated a more sympathetic ethics approval system, and indeed this was negotiated for PgCAP students. For ARC members, however, the situation was not resolved:

....after three months since my ethics application started, I had to abandon the project, as the action research intervention point had passed irrevocably. I'm really disappointed, as this was a flagship project for our institution, providing an excellent chance to show the importance of the student voice in planning and delivery; now it's just a lost opportunity.

\section{Aligning aspirations to changing circumstances}

As my project progressed (over the past two years) my original aims and design had to be modified still further due to changing circumstances, but that opened up new insights and led to the formulation of further questions at an institutional level: Can support frameworks for action research model the protocols of PDP and CPD so that they stimulate a dynamic culture of evidence-based joint practice development? When undertaken with peers acting as 'critical friends', can action research create communities of self-motivated practitionerresearchers who continuously effect improvements in their practice?

My findings from the ARC (limited though they are) indicate that the model itself is worthy of being sustained and enhanced - but in a different way. If we are to achieve personal engagement in CPD combined with institutional development in pedagogic practice, aligning vision, values and practices will go a long way towards overcoming the barriers 
that were encountered by ARC members. I hope that readers will use the lessons learned section on 'what didn't work' to deal with such barriers, or preferably prevent them arising in the first place. On the other hand, we can use 'what works well' in the spirit of Appreciative Inquiry. Combined with a cascading model, it can become the starting point of further cycles and upward spirals of development.

The main recommendation is that managing an effective and collaborative action research process for staff is not far different from facilitating CPD or a PDP process for students. All such developmental processes involve a number of complex social and psychological values as well as practical considerations. Conceptually, a joined-up approach between these factors can turn the simple cycle of plan-do-review into systematic, collaborative enquiry made public - an upward spiral of plan, design, collect data, reflect/evaluate/analyse, record/write/publish, disseminate, share, cascade - then plan a further cycle, and so on.

\section{Flash forward to the future}

If I were evaluating our university's achievements in 2015 I would like to be able to report as follows (and my recommendations are implicit in what follows):

'In the past five years the university's Action Research Programme, directed and supported by senior staff in T\&L and HR, created an extensive research portfolio informing policy and practice across all subject areas. A university-wide programme has incrementally engaged staff by upscaling, enhancing and valuing small-scale, research-related activity so that it is now undertaken collaboratively as cycles and spirals of robust action research, creating a new culture of personal and professional development. The benefits and end-goals of action research concepts are explained in theory, but also promoted and demonstrated as normal, integral (and not optional) professional practice.

Ethics committees meet frequently, not only to consider project proposals but to provide support and help to develop them in a way that is sympathetic to the particular conditions affecting action research. Staff are encouraged to learn by doing and reflecting, and to use peer support, coaching, feedback, individual and 
group guidance provided for action researchers - all in the spirit of Appreciative Inquiry. There is a recognition that the opportunities provided are differentially available and needed by individuals, and they are enabled to engage with them by identifying how their self-MAP maps onto the demands of action research. Since this process of self-mapping is also a central tenet in PDP, it is appropriately rolemodelled and replicated for students.

Working at several levels in a joined-up management framework, an integrated, structured and supported process of PDP, CPD and action research has created a professional community of joint practice development and a 'practitioner research footprint' through dissemination, publication and cascading techniques - influencing PDP, CPD and pedagogy across the HE landscape'.

If such progress were to be made it would be a testament to the powerful legacy of the NARN and the ARC experience.

\section{Acknowledgement}

This paper is an outcome of the National Action Research Network on researching and evaluating Personal Development Planning and e-Portfolio practice project (2007-2010). The project was led by the University of Bolton in association with the University of Worcester and Centre for Recording Achievement, and in national collaboration with the University of Bedfordshire, Bournemouth University and University of Bradford. The project was funded by the Higher Education Academy, National Teaching Fellowship Project strand. More details about the project can be found at: http://www.recordingachievement.org/research/narn-tree.html.

\section{References}

Altrichter, H., Posch, P. and Somekh, B. (1993) Teachers investigate their work: an introduction to the methods of action research. London and New York: Routledge. 
Argyris, C. and Schön, D. (1974) Theory in practice: increasing professional effectiveness. San Francisco: Jossey-Bass.

Atlay, M.T. (2008) University of Bedfordshire education strategy (2008-13): transformational education. Luton: University of Bedfordshire. Available at: http://www.beds.ac.uk/learning/curriculum (Accessed: 29 September 2010).

Atlay, M. (2010) Bridges CETL: final report to HEFCE. Luton: University of Bedfordshire.

Beard, J. (2010) 'Organising small scale action research on a large scale: a case study of the PILS CETL management framework at the Open University', in Atlay, M. (ed.) Creating bridges: a collection of articles relating to the implementation of the Curriculum Review 2008 (CRe8) from practitioners across the University of Bedfordshire. Luton: University of Bedfordshire internal publication, pp. 201-210.

Joyce, B.R. and Showers, B. (2002) Student achievement through staff development. Alexandria, VA: Association for Supervision and Curriculum Development.

Kotter, J.P. and Lawrence, P.R. (1974) Mayors in action. NY: John Wiley \& Sons.

Kramp, M.K. and Humphreys, W.L. (1995) 'Narrative, self-assessment, and the habit of reflection', Assessment Update, 7(1), pp. 10-13.

Kumar, A. (2007) Personal, academic and career development in higher education SOARing to success. London and New York: Routledge, Taylor and Francis.

Kumar, A. (2009a) 'Using assessment centre approaches to improve students' learning', in Nygaard, C., Holtham, C. and Hawthorn, N. (eds.) Improving students' learning outcomes. Frederiksberg, Denmark: CBS Press.

Kumar, A. (2009b) 'SOARing for employability: can assessment centre approaches engage students?' in Atlay, M. (ed.) (2010) Creating bridges: a collection of articles relating to implementing the Curriculum Review 2008 (CRe8) from practitioners across the University of Bedfordshire. Luton: University of Bedfordshire internal publication, pp. 84-103. 
Locke, E.A., Latham, G.P., Smith, K.J. and Wood, R. E. (1990) A theory of goal setting and task performance. Englewood Cliffs, NJ: Prentice Hall.

Maslow, A.H. (1970) Motivation and personality. New York: Harper Row.

Newman, B.M. and Newman, P.R. (2009) Development through life: a psychosocial approach. CA: Wadsworth Cengage Learning.

QAA, UUK, SCoP and CoSHEP (2001, revised 2008) Guidelines for HE progress files. Gloucester: Quality Assurance Agency for Higher Education (UK).

Schön, D. (1991) The reflective practitioner: how professionals think in action. NY: Basic Books.

Senge P. and Scharmer, C. (2001) 'Community action research: learning as a community of practitioners, consultants and researchers', in Reason, P. and Bradbury, H. (eds.) Handbook of action research. London: Sage.

Stenhouse, L. (1981) 'What counts as research?', British Journal of Educational Studies, 29(2), pp. 103-114.

Watkins, J. and Cooperrider, D. (2000) 'Appreciative inquiry: a transformative paradigm, Journal of the Organization Development Network, 32(1), pp. 6-12.

Wenger, E. (1998) Communities of practice: learning, meaning and identity. Cambridge: Cambridge University Press.

\section{Author details}

Arti Kumar's work, as Associate Director of the Centre for Excellence in Teaching and Learning (CETL) at the University of Bedfordshire, has been central in the university's adoption of effective learner-centred pedagogies that connect personal and career development with good learning and employability approaches in higher education curricula. As part of her National Teaching Fellowship project (awarded in 2005) she 
authored the book entitled Personal, Academic and Career Development in Higher Education - SOARing to Success published in 2007 by Routledge Taylor \& Francis. She was awarded an MBE in the Queen's Honours list 2008 in recognition of her services to higher education. She is currently an Honorary Visiting Research Fellow at the University of Bedfordshire and also a Fellow of the National Institute of Career Education and Counselling (NICEC) and of the UK Higher Education Academy. She is an AGCAS Lifetime Achievement Award winner 2010. 